\title{
Les déclinaisons historiques du franco-judaïsme et ses critiques contemporaines. «Peut-on être un juif émancipé ?» (Emmanuel Levinas)
}

À propos de :

LEFF Lisa Moses, Sacred Bonds of Solidarity. The Rise of Jewish

Internationalism in Nineteenth-Century France. Stanford, Stanford University Press, 2006, $327 \mathrm{p}$.

MALINOVICH Nadia, French and Jewish. Culture and the Politics of Identity in Early Twentieth-Century France, Portland, The Littman Library of Jewish Civilization, 2008, 280 p.

SAVY Pierre, SCHREIBER David, (coord.), « Des Juifs contre l'émancipation. De Babylone à Benny Lévy ", Labyrinthe, 28-3, 2007.

\section{Martine Cohen}

\section{OpenEdition Journals}

Édition électronique

URL : http://journals.openedition.org/assr/18203

DOI : 10.4000/assr.18203

ISSN : 1777-5825

Éditeur

Éditions de l'EHESS

Édition imprimée

Date de publication : 1 octobre 2008

ISBN : 978-2-7132-2192-7

ISSN : 0335-5985

Référence électronique

Martine Cohen, «Les déclinaisons historiques du franco-judaïsme et ses critiques contemporaines «Peut-on être un juif émancipé ?» (Emmanuel Levinas) », Archives de sciences sociales des religions [En ligne], 144 | octobre-décembre 2008, mis en ligne le 20 novembre 2012, consulté le 30 avril 2019. URL : http://journals.openedition.org/assr/18203 ; DOI : 10.4000/assr.18203 


\title{
Martine Cohen
}

\section{Les déclinaisons historiques du franco-judaïsme et ses critiques contemporaines \\ “Peut-on être un juif émancipé ? " (Emmanuel Levinas)}

\author{
À propos de : \\ LeFF Lisa Moses, Sacred Bonds of Solidarity. The Rise of Jewish Inter- \\ nationalism in Nineteenth-Century France. Stanford, Stanford \\ University Press, 2006, 327 p. \\ Malinovich Nadia, French and Jewish. Culture and the Politics of \\ Identity in Early Twentieth-Century France, Portland, The Litt- \\ man Library of Jewish Civilization, 2008, 280 p. \\ SAVY Pierre, SCHREIBER David, (coord.), « Des Juifs contre l'émancipa- \\ tion. De Babylone à Benny Lévy ", Labyrinthe, 28-3, 2007.
}

Les débats sur les bienfaits de l'égalité des droits civils accordée aux juifs de France en 1791, ou au contraire sur ses conséquences négatives et le risque de disparition de l'identité juive (ou plus directement sur la supposée visée d' « assimilation » des révolutionnaires) ne concernent pas que les travaux universitaires récents (depuis les années 1970-80); on les retrouve aussi dans les discours des acteurs juifs français eux-mêmes, dès le XIX ${ }^{\mathrm{e}}$ siècle, traduisant leurs luttes pour la redéfinition légitime de l'identité juive en régime d'égalité et de liberté de culte ${ }^{1}$.

Ces débats ont porté (ou portent encore) soit sur les visées (explicites ou non) des promoteurs de l'émancipation des juifs, soit sur les « réponses » des acteurs juifs : que firent notamment les différentes élites juives (rabbins, intellectuels, notables, bourgeoisie parisienne naissante) pour défendre ou promouvoir l'identité juive dans ce nouveau contexte ? Cette seconde perspective, avec sa démarche compréhensive, est aujourd'hui la plus courante parmi les historiens du judaïsme français - c'est le cas des deux études historiques proposées ici par Lisa Moses

1. On sait que cette liberté et cette égalité ne furent pas acquises d'emblée et uniformément pour tous les juifs de France : citons en exemple le "décret infâme " promulgué par Napoléon en 1808 et qui n'a concerné que les juifs d'Alsace (à la suite des protestations des juifs du SudOuest et du Comtat venaissin). 
Leff et Nadia Malinovich. Mais il faut noter l'inflexion de ces travaux, notamment parmi les historiens nord-américains depuis la fin des années soixante-dix, vers une évaluation plutôt positive de l'action de ces acteurs : au contraire de Michaël Marrus (1971), qui critiquait la "politique d'assimilation » de l'establishment juif, l'historienne du Consistoire Phyllis Cohen Albert (1977) défendait la thèse d'une construction identitaire juive nouvelle au XIX ${ }^{\mathrm{e}}$ siècle, utilisant même le terme d' "ethnicité ", alors en vogue depuis une dizaine d'années dans les travaux de sciences sociales aux États-Unis ${ }^{2}$. Ces débats sur l'émancipation peuvent enfin porter sur les processus en jeu et leurs effets, même involontaires, de «dilution» de l'identité juive. C'est le cas de ces «penseurs du retour» ou des critiques de l'émancipation qui font l'objet du dossier de la revue Labyrinthe coordonné par Pierre Savy et David Schreiber.

\section{L'internationalisme juif au service de l'intégration nationale}

À travers son étude de "l'internationalisme juif » au XIX $x^{e}$ siècle, L. M. Leff appelle d'emblée à un "modèle plus nuancé de l'intégration des juifs » (p. 4). Sa thèse centrale lie en effet l'intégration nationale des juifs en France à l'activisme international de leurs élites intellectuelles et politiques pour développer une solidarité juive nouvelle : promouvoir l'égalité des droits pour tous les juifs persécutés dans le monde, selon le modèle individualiste et confessionnel français (les principes de liberté et d'égalité religieuse), était en effet un moyen de mieux assurer leurs propres droits en France même. L'auteure souligne par là même l'engagement des juifs dans la construction d'un "État laïcisé » ${ }^{3}$, et l'importance de la dimension internationale de cette construction - notamment à travers la "mission civilisatrice » de la France dans ses sphères d'influence et dans l'Algérie coloniale, mission séculière que les juifs souhaitaient substituer à l'idée de " mission catholique ».

Si l'internationalisme juif est ainsi un produit (et un support) de l'intégration nationale en France, c'est que les transformations de l'identité juive en France n'ont pas été, nous dit l'auteur, dans le sens de la "privatisation" que l'on souligne habituellement. Bien au contraire, des élites religieuses, mais aussi intellectuelles et politiques, qui constituaient de fait un noyau parisien, ont fait du judaïsme une composante centrale et publique de l'appartenance citoyenne à la France. Les uns ont réutilisé les termes de la tradition religieuse pour légitimer cette appartenance nouvelle, tandis que d'autres sécularisaient ces termes (et notamment celui de "solidarité ») pour appeler la France à promouvoir ses «idéaux de 89 » sur la scène internationale. L'identité juive aurait été ainsi au

2. Voir l'étude de Ph. C. Albert (1977) sur la création du système consistorial.

3. Cette formule me semble la plus neutre pour traduire l'expression State secularism utilisée par l'auteur. On pourrait aussi parler de « laïcisation de l'État» ou de «laïcité de l'État". Je reviendrai plus loin sur ce point. 
cœur d'un discours public des juifs. Elle aurait été même, du fait de sa sécularisation et de son association aux valeurs libérales par certains intellectuels et hommes politiques juifs, un symbole d' "universalité » pour des militants libéraux ou les socialistes utopiques (les Saint-Simoniens, qui comptaient dans leurs rangs de nombreux juifs plus ou moins assimilés ou même convertis).

La vision "nuancée " de l'intégration appelle ainsi naturellement une vision plus complexe, et d'abord plurielle, de la nouvelle identité juive française liée à cette intégration. Religieuse, sécularisée... ou même assimilée, cette identité juive fut loin de s'effacer (dans la sphère privée) ou de se diluer - comme le dénoncent certains critiques contemporains des Lumières et de l'émancipation. Elle s'est recomposée (identity was reshaped), de la même manière d'ailleurs que pour d'autres sous-groupes de la France post-révolutionnaire - Bretons, ouvriers, paysans.

L'ouvrage est composé de six chapitres qui établissent, de manière chronologique, les « racines » de l'internationalisme juif, puis son émergence et son développement dans la seconde moitié du XIX ${ }^{\mathrm{e}}$ siècle, puis sa mise en échec devant la montée d'un nouvel antisémitisme, à partir des années 1880, alors même que les idéaux de la République triomphaient.

La subordination du judaïsme à l'État opérée par Napoléon, nous dit Leff dans un premier chapitre, ne doit pas être confondue avec une quelconque privatisation du judaïsme, bien au contraire. Au plan idéologique en effet, le judaïsme était mobilisé, réinterprété, pour apporter une légitimation religieuse à l'adhésion des juifs à la France, mettant en avant «l'amour des frères » français au-dessus de toute autre proximité avec des juifs d'autres pays, et promouvant des valeurs universelles. Au plan institutionnel ensuite, le système consistorial était intégré à l'administration d'État, lui servant notamment de relais dans ses fonctions de police (les consistoires locaux devaient contrôler l'immigration des juifs dans l'Empire) ou comme institution d'éducation pour promouvoir une morale civique. Le judaïsme devenait ainsi une composante centrale de la citoyenneté française des juifs. Mais bien des mesures discriminatoires maintenaient leur statut de corporation lié à l'Ancien régime et des leaders juifs durent lutter, en nouant des alliances avec des hommes politiques libéraux de la Restauration et en se réclamant des principes de la Charte de 1814, pour obtenir leur abolition (chap. 2). Leurs succès furent inégaux : certes le " décret infâme » de 1818 ne fut pas reconduit, mais le serment more judaïco ne fut aboli que tardivement, en 1846 seulement. Les premières alliances nouées allaient cependant être consolidées ensuite et permettre de diffuser la vision positive d'un judaisme sécularisé, " oriental » ou « cosmopolite ", notamment au sein du mouvement des Saint-Simoniens ou parmi des auteurs romantiques (chap. 3).

C'est dans la seconde moitié du XIX ${ }^{e}$ siècle que va se déployer une nouvelle solidarité juive internationale, toujours référée par ses promoteurs à la « mission 
civilisatrice » de la France (chap. 4). L'affaire de Damas, en 1840, sera la première occasion d'un tel appel de leaders juifs, le français Adolphe Crémieux (alors vice-président du Consistoire central) et l'anglais Sir Moses Montefiore, à l'aide de la France pour laver des juifs syriens de l'accusation de meurtre rituel de deux frères capucins. La protection apportée par la France à des congrégations religieuses du Levant sera à nouveau mise en balance lors de la guerre de Crimée (1853-56), cette fois avec la demande de droits égaux pour les juifs de l'empire ottoman. Mais c'est dans l'Algérie coloniale que les responsables consistoriaux (rabbins et laïcs) vont déployer leurs efforts avec le plus d'efficacité pour apporter les bienfaits de l'émancipation aux juifs d'Algérie : la fin de leur statut de corporation impliquera alors, aussi, la fin de leur autonomie communautaire et leur prise en charge (forcée) par le rabbinat français métropolitain, dans un esprit de " civilisation »; leur accession à la citoyenneté française ensuite, grâce au décret d'Adolphe Crémieux (en 1870, il est alors ministre de la Justice d'un gouvernement républicain provisoire), signera tant l'abolition définitive de leur statut personnel que leur distinction définitive des autres "indigènes ", musulmans ${ }^{4}$. La création de l'Alliance israélite universelle en 1860 (chap. 5) marquera la sécularisation et la modernisation de la notion de "solidarité " entre juifs. Alors que celle-ci était imposée par la tradition et impliquait, sous le régime de corporation, un lien d'interdépendance entre tous les membres d'une communauté juive face à ses obligations et ses dettes - le groupe juif étant lui-même dans un rapport d'exclusion et d'inégalité par rapport à son environnement chrétien - la solidarité promue par l'Alliance s'appuyait sur l'adhésion volontaire à une association promouvant une cause libérale et universelle, et s'inscrivait dans une société à laquelle les juifs participaient pleinement, sur un pied d'égalité avec les autres citoyens. Le réseau international de ses Comités locaux (établis pays par pays, mais toujours soumis à une direction siégeant à Paris et rédigeant son Bulletin en français) et la multiplication des écoles primaires de l'Alliance dans tout le bassin méditerranéen, allaient être les instruments d'une telle solidarité (encore en œuvre jusqu'à aujourd'hui dans certains pays). Mais ce déploiement international donna prise ensuite à l'élaboration d'un "mythe " pernicieux, celui d'une " puissance juive » ou d'un « complot juif » mondial (chap. 6), porté par un antisémitisme nouveau et qui allait imposer aux juifs français une prudence nouvelle dans leur expression publique.

En conclusion, Leff insiste donc: les leaders juifs n'ont ni abandonné leur identité juive ni reconduit une identité de type "tribal». En identifiant leur religion à une vertu civique et en remplaçant leurs institutions communautaires par des institutions étatiques (state agencies), ils ont aussi inscrit l'égalité religieuse à l'agenda des républicains, contribuant activement à la laïcisation de

4. Les juifs furent très peu nombreux à renoncer à ce statut personnel lors de la proposition de citoyenneté française par choix individuel formulée par le sénatus-consulte de Napoléon III en 1865 . 
l'État. Mais leur internationalisme n'était pas sans risque ou contradiction, et ceux qui y ont travaillé (les leaders de l'AIU en particulier) sont devenus les premières cibles de l'antisémitisme de la fin du XIX ${ }^{\mathrm{e}}$ siècle.

L'intérêt de l'ouvrage réside d'abord dans l'originalité de sa thèse - avec son caractère contre-intuitif: l'intégration nationale des juifs de France passait par le déploiement d'une politique de solidarité internationale avec leurs frères juifs persécutés dans le monde. Il réside aussi dans les analyses proposées de la sécularisation-modernisation de la notion de "solidarité ", et dans la description détaillée des combats des leaders juifs parisiens pour mettre fin aux discriminations persistantes légalement imposées aux juifs jusqu'au milieu du XIX ${ }^{e}$ siècle. Est fort intéressante enfin la démonstration des liens étroits entre plusieurs des objectifs ou des valeurs politiques des républicains : libéralisme, laïcité, et politique étrangère d'extension de l'influence française, dont fit partie la politique coloniale en Algérie, appuyée sur une idéologie de diffusion de la " civilisation ".

On peut cependant regretter que l'auteur n'ait pas pris connaissance, pour parler de la laïcisation de l'État (la promotion d'un state secularism), des travaux de Jean Baubérot sur l'histoire de la laïcité française. En prenant en compte notamment la notion de "premier seuil de laïcisation ", elle aurait peut-être pu éviter de poser - pour la contredire - une image commune de la laïcité comme irréligion ou comme reflux de la religion dans la sphère privée. Dès le début du $\mathrm{XIX}^{\mathrm{e}}$ siècle, en effet, et jusqu'aux années 1850-1860, l'idée d'une utilité sociale de la religion pour préserver la morale commune et promouvoir le civisme, ainsi que l'association des quatre cultes reconnus à l'État, ont clairement donné une "publicité » à la religion. Cette précision permettrait alors de distinguer plusieurs aspects dans la notion de "privatisation " : l'invisibilité dans l'espace social commun d'une part, l'individualisation de l'appartenance religieuse et la dissociation entre celle-ci et la citoyenneté d'autre part (dissociation pouvant mettre fin aux discriminations à fondement religieux). Ce deuxième aspect, l'individualisation et "l'indistinction " politique des appartenances implique bien une forme de privatisation qui était recherchée par les leaders juifs de l'époque. Si ceux-ci ne s'interdisaient pas pour autant d'afficher leur identité juive (aspect de la visibilité sociale), c'est parce qu'ils associaient celle-ci à des valeurs universelles pouvant participer d'une morale commune et soutenir leur civisme français. Pourrait-on parler à leur propos de recherche d'une "religion civile ", ou seulement pour ceux d'entre eux qui s'engagèrent aux côtés des Saint-Simoniens à la recherche d'une nouvelle religiosité pour remplacer le catholicisme traditionnel ? Les descriptions et les analyses proposées par l'auteur peuvent ainsi alimenter toute une discussion sur le processus non linéaire de laïcisation de l'État français, entre établissement d'un pluralisme religieux progressivement plus égalitaire et séparation complète de l'État et des Églises, en passant par les tentatives de « religion civile » - toutes finalement écartées, si l'on suit la thèse de Baubérot. 
I46 - ARCHIVES DE SCIENCES SOCIALES DES RELIGIONS

\section{Le "Réveil " des années 1920 : une conception ethnoculturelle de l'identité juive}

Dans une étude historique magistrale sur la vitalité associative et artistique des juifs de France au cours des années vingt (qualifiée par certains acteurs de l'époque de "renaissance " ou de "Réveil »), N. Malinovich appelle elle aussi à "réévaluer [la thèse de] l'assimilation". Tous les militants d'une affirmation identitaire juive plus ouverte (publique), nous dit-elle, partageaient en effet une compréhension ethnoculturelle du judaïsme, que celle-ci s'exprime à travers l'activisme pro-sioniste (soutien à la création d'un foyer national juif en Palestine pour sauver les juifs persécutés dans le monde) ou à travers une religiosité soulignant le particularisme juif - sans opposer cependant celui-ci à l'universalisme. De la même manière, Malinovich appelle à "réévaluer le franco-judaïsme ". Si certains acteurs du Réveil juif des années vingt critiquaient les générations précédentes, d'autres s'inscrivaient au contraire dans leur continuité et considéraient que l'émancipation et la citoyenneté avaient ouvert la voie à une nouvelle vitalité juive, ou même à l'idée d'un État autonome. Remarquons que ces appels à la " réévaluation », en reprenant les débats des acteurs eux-mêmes, s'adressent implicitement et tout autant aux chercheurs d'aujourd'hui.

L'étude de Malinovich s'inscrit dans la suite de celle de Leff. Celle-ci s'arrête avant l'affaire Dreyfus, celle-là commence par l'analyse de l'impact de cette affaire sur les juifs de France. Alors que Leff évoque la nouvelle prudence des élites juives dans leur discours public, Malinovich souligne le phénomène inverse - qui certes ne concerne pas l'ensemble des juifs de France - de "politisation » de l'identité juive, au-delà même du combat contre l'antisémitisme, avec un engagement juif au sein de la gauche (l'écrivain André Spire et l'homme politique Léon Blum seraient les figures emblématiques de cette "conversion »).

Malinovich décrit de manière très documentée et vivante ces juifs français occupés à redéfinir leur judaïsme et ses rapports avec leur identité française, dans une société où ils jouissent d'une pleine égalité politique. Si cette vitalité concerne de fait une minorité parmi les juifs de France, c'est une minorité active et influente, tant dans la communauté juive (pas seulement parisienne) que dans la société française. Après une "première vague " de production littéraire mais aussi de création de revues et de mouvements de jeunesse avant la guerre de 1914-18, le dynamisme créatif reprend de plus belle dans les années vingt : revues (dont celles des mouvements de jeunesse), clubs de discussion, productions littéraires, musicales et picturales (l'École de Paris à Montparnasse), associations politiques de soutien au sionisme ou de lutte contre l'antisémitisme (la LICA). Le champ religieux est lui aussi l'espace de débats et de conflits : après sa création en 1907 (rendue possible par la Séparation des Églises et de l'État en 1905), l'Union Libérale Israélite (ULI) a suscité en face d'elle l'émergence de militants plus traditionalistes, des rabbins réunis autour de la revue Foi et Réveil (fondée en 1913, en réponse à celle de l'ULI, Le Rayon) et du mouvement Chema Israël 
(créé en 1919). Puis l'atténuation des réformes radicales de l'ULI et un certain rapprochement avec le Consistoire (obtenu grâce aux efforts d'Aimé Pallière ${ }^{5}$ ), ont mis en exergue plutôt les conflits entre religieux et partisans d'une identité juive plus séculière (culturelle ou nationale), les premiers accusant les seconds de perdre la substance même du judaïsme, tandis qu'en retour, ces derniers accusaient les premiers de "réduire » celui-ci à une religion ${ }^{6}$. Du côté d'un judaïsme culturel ou ethnique, on trouve notamment l'Union Universelle de la Jeunesse Juive (fondée en 1921 à Salonique avant d'être transférée à Paris par ses fondateurs en 1923) et le mouvement scout des Éclaireurs israélites de France (EIF), qui s'en rapprochera d'une certaine manière, sous l'influence de l'écrivain Edmond Fleg ou du mouvement sioniste. De fait, les EIF deviendront un lieu important d'ouverture à la pluralité des manières d'être juif ${ }^{7}$. La création littéraire (le poète André Spire ou l'écrivain salonicien Albert Cohen, par exemple) et la multiplication des traductions du yiddish (Israël Zangwill, Chalom Aleichem, ...) ou de l'hébreu (Bialik) font connaître les juifs d'autres régions du monde dans leur diversité culturelle (ceux d'Europe de l'Est, de l'empire ottoman ou d'Afrique du Nord), mais aussi la diversité de leurs options identitaires : des nationalistes farouchement antireligieux aux orthodoxes antisionistes.

Parmi les facteurs majeurs de déploiement de ce « Réveil » dans sa dimension ethnique ou culturelle, Malinovich souligne l'influence du mouvement sioniste, qui compte en France plusieurs associations de soutien et quelques sympathisants au sein du leadership institutionnel juif (Consistoire, AIU), malgré l'opposition idéologique de fond de celui-ci à toute conception nationale du judaisme. Dans cette perspective politique également, l'affichage des droits des minorités nationales lors de la Conférence de la Paix à Versailles en 1919 - parmi lesquelles s'inscrivirent les juifs de plusieurs pays d'Europe orientale - joua en faveur d'une accommodation des esprits à cette nouvelle vision. L'auteure souligne également l'influence des immigrés juifs d'Europe de l'Est, ceux arrivés jeunes en France ou leurs enfants surtout. En racontant leurs échanges et leurs multiples productions communes avec les juifs autochtones, elle souhaite contrebalancer l'image courante d'une opposition tranchée entre ces deux secteurs de la population juive.

5. A. Pallière fut l'infatigable militant du rapprochement entre Libéraux et traditionalistes, mais aussi d'un sionisme plus religieux ; et cela, malgré sa situation de "noahide " : catholique spirituellement proche du judaïsme, il ne considérait pas nécessaire de se convertir puisque, selon la tradition juive même, le respect des sept lois de base de l'humanité (" noahiques ": de Noé) est suffisant au salut de tout homme.

6. Dans le domaine religieux, il faut mentionner aussi les débuts d'un mouvement interconfessionnel et le contexte favorable d'émergence d'un catholicisme libéral, autour des mouvements d'Action catholique ou de syndicalisme chrétien, ou aussi de l'École de Lyon, branche française d'un courant moderniste.

7. L'historien de ce mouvement, Alain Michel, estime que cette idéologie pluraliste a constitué un principe fondateur de la vie associative juive d'après-guerre; nombre de nouveaux leaders institutionnels juifs sortiront de ses rangs (noté par N. Malinovich dans sa conclusion). Cf. A. Michel, 2003. 
Elle rappelle aussi l'émergence, dans ces mêmes années, d'une littérature régionaliste et des études folkloristes comme discipline ${ }^{8}$.

Mais, pour elle, le facteur le plus décisif fut le "sentiment de sécurité » éprouvé par les juifs de France : ce désir d'être "plus juif » (elle reprend ici une expression d'Albert Cohen), fut "le produit du succès de l'intégration » (p. 201). La France des années vingt est, à cet égard, bien différente de l'Allemagne. L'auteure nous propose plusieurs comparaisons fort intéressantes des contextes nationaux de ces deux pays et des deux mouvements de regain culturel et ethnique juif. Si celui qui se déploie en France émerge d'une situation positivement perçue où l'antisémitisme semble en déclin ${ }^{9}$, celui des juifs allemands s'inscrit dans un contexte tendu de montée de l'antisémitisme et d'exclusion sociale croissante des juifs; d'où la tendance de ceux-ci à créer leurs propres associations séparées, alors que nombre de non-juifs participent à la vie de plusieurs associations juives françaises. Si certains juifs français critiquent la volonté d' "assimilation" de leurs prédécesseurs, d'autres se disent au contraire pleins de gratitude à l'égard de leur travail de réinterprétation et de diffusion d'un judaïsme modernisé ; tel n'est pas le cas en Allemagne, où « l'illusion » des parents sur leur « intégration » est dénoncée et raillée, poussant les juifs à opposer la Judentum à une Deutschtum qui les excluait. L'émergence d'un nationalisme de type ethnique en France aura alors des conséquences beaucoup moins brutales qu'en Allemagne, même si l'essentialisation de la différence juive (souvent promue par les juifs eux-mêmes) se révèlera progressivement ambivalente et même dangereuse ${ }^{10}$.

Le "Réveil » des juifs français atteste ainsi d'une nouvelle compréhension ethnoculturelle du judaisme, dans laquelle l'auteure inclut, non seulement les partisans d'un "nationalisme juif » en Palestine, mais aussi les militants d'une nouvelle religiosité soulignant le particularisme juif. Produit d'une intégration réussie, il résulta autant des coopérations que des conflits d'interprétations entre juifs. Cette vitalité religieuse et culturelle aurait été, selon Malinovich, «le phénomène d'une génération ", plus occupée de recherche personnelle que soucieuse de continuité et de transmission (p. 238), un phénomène minoritaire, dont seule perdurera l'influence des Eclaireurs israélites de France et du Club Macabi (sportif). L'auteur affirme cependant dans sa conclusion que cette première expérience d'une forme ethnoculturelle de franco-judaïsme sera renouvelée après guerre, dans un contexte de transformations nombreuses de la communauté. On discutera plus loin cette vision prospective et la caractérisation de cette première expérience ethnoculturelle comme "franco-judaïsme ».

8. N. Malinovich renvoie sur ce point à l'étude d'Anne-Marie Thiesse, 1988.

9. Rappelons notamment la loi qui facilita le processus de naturalisation, en 1927, dont bénéficièrent de nombreux juifs immigrés.

10. L'auteure cite quelques auteurs révélateurs de « cette ère où la distinction entre célébration de la différence ethnique et renforcement des stéréotypes racistes était facilement estompée" (p. 200). 


\section{L'émancipation des juifs : proposition de rêve ou marché de dupes?}

Alors que Leff et Malinovich décrivent par le menu, documents d'archives à l'appui, les constantes réinterprétations et recompositions de l'identité juive ${ }^{11}$, plusieurs des auteurs du dossier de la revue Labyrinthe (ou des auteurs dont la pensée est analysée) se montrent fondamentalement critiques de l'émancipation des juifs et plus généralement des Lumières, ou au moins ambivalents à l'égard de ses conséquences, surtout en France ${ }^{12}$.

Le contexte de leur interrogation est marqué par le climat particulier des années deux mille en France ${ }^{13}$ : "une série de faits ", certes de "tailles différentes " qui provoque chez eux une "stupeur" ou un "doute ", alors même qu'ils ont été formés «dans la conviction d'une positivité de la Révolution française, de la laïcité et de l'universel »(p. 106). Ce contexte est tout à la fois intellectuel (la publication en 2003 de l'ouvrage du linguiste Jean-Claude Milner sur Les Penchants criminels de l'Europe démocratique, ou la critique radicale des Lumières par le philosophe Benny Lévy) et factuel : les nombreux incidents antijuifs des années 2000 auraient conduit certains juifs à s'interroger sur "l'avenir des Juifs de France » (c'est le titre choisi par le sociologue Shmuel Trigano pour son ouvrage publié en 2006), ou sur la "capacité protectrice » de l'État français (on reviendra plus loin sur cette question de l'historien Pierre Birnbaum), et enfin les nouveaux débats sur la laicité et l'intégration, ainsi que la présence de l'islam, dont le développement en France « enrichit la question de la "diversité" et (...) pose des questions qui ne sont pas sans parenté avec celles que l'on a affrontées ici» (p. 107) ${ }^{14}$. Bref, tout en reconnaissant l'existence d'un

11. Mais il est vrai que toute analyse comporte une part d'interprétation, qui explique au moins partiellement leur " différend " avec l'analyse critique de la "politique assimilationniste " de M. Marrus par exemple.

12. Sur les huit articles du dossier coordonné par P. Savy et D. Schreiber, on concentrera notre analyse sur quatre d'entre eux qui nous semblent centraux : l'entretien avec P. Birnbaum ( "Descartes ou le knout ? ", pp. 11-23); H. Hermant: "Refus des Lumières : les penseurs du retour face au danger de la dissolution de l'identité juive " (pp. 25-38) ; D. Nadjari : " L'émancipation “à marche forcée” des Juifs d'Algérie » (pp. 77-89) ; P. Savy, D. Schreiber : «Émancipation(s) ?" (article qui clôt le dossier). Les quatre autres articles sont : Yehezkel Lévy : "L'exil de Babylone : les sources traditionnelles et la question de l'émancipation " (pp. 39-51); Franck Lemonde : «Un Aufklärer entre deux feux : Mendelssohn (1729-1786)» (pp. 53-62) ; Pierre Savy : "Le tsar, l'empereur et le Roi des rois : remarques sur les géographies de l'existence » (pp. 63-75) ; Aurélia Smotriez : « Émancipation, sionisme et éducation : aux origines du système scolaire israélien » (pp. 91-103). Les deux premiers de ces articles seront pris en compte ponctuellement dans la discussion.

13. On reprend ici les termes des coordinateurs du dossier dans leur article de clôture : "Émancipation(s)?".

14. De fait, aucun article du dossier ne porte sur ce thème de l'islam, censé poser les mêmes questions mais " avec un important décalage chronologique ». 
« renouveau de la vie juive en France depuis 1945 »(p. 115), Savy et Schreiber n'en pensent pas moins que la " question juive » demeure posée, qu'ils identifient comme étant celle de «la survie » du peuple juif et, pour certains, celle de son « incompatibilité » avec la modernité, politique ou philosophique.

\section{La fin d'une « légende dorée » et d'autres « mythologies »...}

"La légende dorée de l'émancipation a donc vécu ", écrit Héloïse Hermant dans son analyse des "penseurs du retour " [à la tradition juive] (p. 26), constat que nuancent les coordinateurs du dossier : cette "légende dorée » dominerait encore au plan officiel (les commémorations, les manuels scolaires), mais au plan intellectuel, sa doxa serait considérablement affaiblie. Plus factuellement, Birnbaum met en cause la "mythologie " d'un Napoléon "libérateur des Juifs ${ }^{15}$ et celle de l'acceptation facile par les juifs de France des consistoires créés autoritairement par l'empereur pour être l'instrument de leur "normalisation " (pp. 14-15). Il s'agit donc de repenser, soit les termes et les conséquences de l'émancipation des juifs de France, soit certains de ses "moments", soit encore et plus généralement l'ensemble du projet d'émancipation et le rapport des Lumières aux juifs (ce que font les «penseurs du retour » ou d'autres, comme Milner, qui ne sont pas dans une telle démarche religieuse).

Birnbaum souligne dans son entretien cette contradiction incroyable entre "une émancipation rêvée, extraordinaire" en France, et ses "conséquences inverses » ou ses "effets pervers " : l'appauvrissement de la vie juive, « une certaine stérilisation de la pensée » (p. 19). En s'interrogeant sur les conditions ou les contextes qui sont (ou ont été) au contraire favorables au maintien d'une vie juive, il cite la Russie persécutrice, l'Allemagne dont l'identité «fondamentalement chrétienne, plus éloignée des Lumières " et la société hostile ont incité à "l'inventivité juive ", ou encore les États-Unis avec leur «esprit démocratique " acceptant le pluralisme et le localisme, à la manière d'un Empire (d'ailleurs ce pays "n'est pas une nation », précise l'auteur). Dans leur article commun, Savy et Schreiber posent que "l'émancipation française demeure un des points centraux de la question juive occidentale » (p. 110). Deux raisons principales à cela : d'une part l'ambiguiité du projet révolutionnaire lui-même, qui signait la fin du "Retour à Sion » mais aussi la scission définitive du théologique et du politique - les deux auteurs verraient donc là deux éléments centraux de l'identité juive ; d'autre part le "découpage en domaines ", cette confessionnalisation qui réduisit le judaïsme à un "culte » alors que cela n'aurait aucun sens en droit rabbinique, et par laquelle l'institution consistoriale fut établie autant pour "protéger " que

15. Puisque c'est une citation de son entretien, je conserve ici la graphie avec majuscule du terme "Juif ». Les critiques qu'il développe dans un ouvrage consacré récemment à l'analyse $\mathrm{du}$ "moment napoléonien " (L’Aigle et la Synagogue..., 2007) et qui sont reprises dans l'entretien qui ouvre le dossier, n'impliquent pas chez lui un refus de fond de l'émancipation. 
pour imposer une «émancipation par le haut " (l'expression est de Birnbaum), autant dire une uniformisation et une assimilation dont " les mondes alsacien puis algérien feront les frais » (p. 112).

\section{... et l'impossible abandon d'un modèle français ?}

Pourtant, malgré ces critiques du modèle français et les mérites reconnus à d'autres modèles (états-unien ou allemand), la France républicaine reste un recours aux yeux de ces trois auteurs. Pour Birnbaum, l'État français serait certes le destructeur des cultures collectives par son "ambition de construire un absolu rationnel " (c'est la face "État libérateur ») et cependant le seul protecteur face à une société qui peut être «folle, violente, agitée de guerres civiles ». Pour Savy et Schreiber, "le modèle républicain parait encore préférable à tout affaiblissement qui entraînerait le surgissement d'un communautarisme incontrôlable " (p. 116) ; face à « la tempête » d'un « antijudaisme renouvelé », eux aussi parlent de "défendre cet espace commun » plutôt que « risquer le déchaînement des forces obscures» (p. 116). Et contre « les plus hardis des Juifs "républicains" (qui) réclament l'inscription du droit à la différence culturelle dans la Constitution ", ils semblent préférer la "difficile liberté » (ils reprennent ici le titre d'un livre de Levinas), une " position stimulante mais instable, entre assimilation et refus ». Ainsi selon eux, «l'optimisme permet de juger possible, quoique instable, une existence juive en diaspora » (p. 116). S'ils soulignent alors l'importance du retour à "l'étude " des textes de la tradition (centre névralgique de l'identité juive, selon les penseurs radicaux comme Lévy ou le sociologue Trigano), ils n'en concluent pas moins que le «logico-politique n'est peut-être pas aussi liquidé qu'on le dit. L'État existe encore, le concept de nation est à interroger, ceux de peuple et de souveraineté aussi (...) » (p. 123).

On peut se demander pourquoi un tel « retour » (si l'on peut ainsi emprunter ce lexique ici quasi-religieux) au «modèle républicain ». Pourquoi l'analyse historique ou philosophico-politique opère-t-elle tout d'un coup ce brusque rétablissement sur une position pourtant critiquée ? Les « fondamentaux » de la culture politique française (un État fort, avec ses bons et ses mauvais côtés) ne seraientils en aucune manière transformables, au point qu'il faille forcément revenir au modèle " pur " ? C'est ce que semble admettre Birnbaum, non sans laisser subsister une interrogation ( «La France n'est toujours pas prête - enfin, l'est-elle ? à passer à un autre type de logique, une logique sociale, du bas et non pas du haut ", p. 21). Ne peut-on observer aujourd'hui une situation plus nuancée et nouvelle, en France, où existe une certaine acceptation sociale (si ce n'est une reconnaissance politique) de la diversité des cultures - ce que des sociologues comme Michel Wieviorka appellent un «multiculturalisme à la française "même si cette acceptation est contrariée, ces dernières années, par des «crispations » identitaires et nationales ? Mais peut-être ce brusque retour au "modèle républicain » relève-t-il de la « schizophrénie » du juif français - ou plutôt alors : 
du juif ET français - celle dont parle Birnbaum à propos de ses travaux de recherche, bien démentie cependant par ses publications depuis plus de dix ans ${ }^{16}$ ?

\section{Une critique plus radicale de l'émancipation et des Lumières}

Hélö̈se Hermant pose d'emblée une utile distinction parmi les critiques des Lumières, ce «courant de pensée radical » qui s'exprime depuis deux décennies et qui est animé par «de purs produits de la culture universitaire française ${ }^{17}$. Certains sont ceux qu'elle qualifie de "penseurs du retour " (essentiellement Benny Lévy et Emmanuel Levinas), d'autres rejoignent par leur radicalité les penseurs du retour stricto sensu mais sans partager leur démarche religieuse (Milner), d'autres encore ne récusent pas les Lumières mais déplorent qu'elles n'aient pu enrayer la montée d'un Mal dont les sources remontent au moyen âge (l'historien Georges Bensoussan, $2006{ }^{18}$ ) ; enfin d'autres interagissent avec ces penseurs mais sans forcément construire un système, en soulignant les ambiguités du projet de la modernité : l'auteur cite ici le sociologue Shmuel Trigano (2006), le linguiste Henri Meschonnic (2001) ou l'historienne Monique-Lise Cohen (1992). Elle ne revient sur ce dernier groupe qu'à la fin de son article, concentrant ses analyses sur les trois penseurs du retour qu'elle a identifiés comme tels : Levinas, Lévy, Milner.

$\mathrm{Au}$ cœur de leur critique, la dénonciation d'une "émancipation porteuse d'assimilation " (p. 27). Levinas fut le premier à poser la question «Peut-on être un Juif émancipé ? » au lendemain de la guerre des Six Jours, citant trois facteurs majeurs qui exacerbent les tensions et les contradictions de la "configuration de l'émancipation » : la Shoah, la création d'Israël, et la laïcité française (1963) ${ }^{19}$. $\mathrm{Si}$, pour Levinas, « les Juifs ne peuvent accepter l'universel des Lumières, sous peine de se renier » (p. 32), c'est que celles-ci érigent la raison et son autonomie - pensée auto-engendrée et donc "christique »- en pierre de touche de la liberté et de l'universalité, au contraire des juifs qui ont adopté le principe hétéronome " obéir avant de comprendre " et qui opposent une singularité incarnée par le visage d'autrui à la conceptualisation abstraite (celle-ci, par sa généralisation, réduirait toujours l'autre au même). Pour Milner, l'émancipation-assimilation serait la forme insidieuse de "solution » au "problème juif », qu'il met en regard de l'extermination nazie, cette forme brutale d'une visée plus fondamentale de

16. «L’Université est généralement très rétive pour aborder ce type de sujets [particularistes] », p. 22.

17. «Refus des Lumières : les penseurs du retour face au danger de la dissolution de l'identité juive » (p. 25).

18. Selon H. Hermant, Bensoussan récuserait la modernité mais en gardant les Lumières comme référent positif face à l'obscurantisme, lorsqu'il reconstruit les généalogies meurtrières d'une Europe antisémite (note 1, p. 25).

19. La réédition de 1984, à la Librairie générale française, comporte des textes postérieurs à 1963. 
l'Europe démocratique : sacrifier les juifs sur l'autel de l'Universel. Pour Lévy, les Lumières, dont " la rationalité [logos] implique la laïcisation »(p. 34), seraient fondamentalement chrétiennes : la séparation du religieux et du politique serait un geste christologique - le «meurtre du Pasteur », alors que la parole juive reste nouée à la sagesse des maîtres - et transformerait la religion en " confession cantonnée à la sphère du privé et à laquelle on adhère parce qu'on y croit » (pp. 34-35). Ainsi le régime politique issu des Lumières (l'État-nation) serait criminel non pas au sens d'assassin (Milner) mais parce qu'il « pousserait au suicide par aliénation " : "la laïcité rend le Juif étranger à lui-même parce qu'elle le conduit à son insu à parler un langage qui trahit le sien » (p. 34).

Pour Levinas comme pour Lévy, une seule solution : le retour à l'étude, et le refus d'entrer dans les catégories occidentales de "nation ", "État ", " confession ». Et si Levinas imagine qu'on puisse être "un Juif émancipé si l'on sait maintenir l'équilibre fragile entre engagement et désengagement» (p. 38), pour Lévy, ce n'est qu'en "étranger " et non en "juif » qu'il peut accepter d'obéir aux lois de l'État français : " on ne peut être juif et français» (p. 36). Ainsi Lévy radicalise-t-il le refus lévinassien du politique : seul un «mouvement de repli du monde " (p. 36) peut permettre au juif de se consacrer à l'accomplissement des commandements divins. Il faut préserver « la Loi » contre tous ces « droits » que l'homme veut s'arroger ${ }^{20}$.

\section{Le colonialisme comme avatar de la "civilisation": une critique indirecte des Lumières?}

L'étude de David Nadjari sur "l'émancipation “à marche forcée " des juifs d'Algérie n'est pas une critique frontale de l'émancipation, mais elle semble bien en être une condamnation indirecte, par l'analyse critique de son imposition forcée à des populations qui n'ont pu que résister passivement, avant qu'émerge une élite locale se ralliant au projet importé ${ }^{21}$. Pour les juifs de France, il s'agissait de reproduire en Algérie le même processus de modernisation religieuse mis en œuvre pour eux-mêmes, notamment en traçant une frontière étanche entre appartenance civile et religieuse, et en reléguant le religieux dans la sphère privée.

20. L'analyse des sources traditionnelles sur la question de l'émancipation (article de Y. Lévy), à partir des livres de Daniel et d'Esther dans la Bible, semble bien " confirmer " cette position de refus du politique ; cependant l'insistance renforcée sur le respect des commandements, qui est une manière de limiter les contacts avec la société environnante et donc le risque d'assimilation, ne semble pas aller jusqu'à appeler à un tel repli du monde. À l'opposé, un Mendelssohn (article de F. Lemonde) propose une séparation du temporel et du spirituel pour permettre une vraie liberté de religion, une tolérance ouverte tant à la pluralité des voies religieuses qu'à la liberté spirituelle de chacun dans chaque religion; d'où son refus de toute excommunication (herem) qui serait, selon l'auteur, la seule innovation religieuse de Mendelssohn, celui-ci restant sur le fond extrêmement ferme en matière de respect des commandements divins.

21. "L'émancipation "à marche forcée" des Juifs d'Algérie et le décret Crémieux" (p. 77-89). 
C'est donc un véritable « lobby consistorial » qui a fait pression sur l'État monarchique français, secondant volontiers l'État dans son projet colonial. L'auteur décrit les étapes de la francisation et de la perte d'autonomie locale : entre 1830, lorsque les représentants traditionnels de la nation juive auprès du souverain musulman (les muqaddam) sont placés sous autorité militaire, et 1870, date du décret Crémieux octroyant aux juifs d'Algérie la pleine citoyenneté française, il y a eu successivement dissolution des tribunaux rabbiniques (1842), création de consistoires locaux (1845-47) puis leur mise sous tutelle du Consistoire central métropolitain, et en 1865 avec le sénatus-consulte de Napoléon III, proposition d'accès individuel à la citoyenneté, sous réserve d'abandon volontaire du statut personnel - ce que les juifs ont refusé dans leur grande majorité, associant à cette démarche, comme les musulmans, l'idée d'apostasie.

L'auteur souligne fort justement deux différences majeures entre ce processus d'émancipation et celui qui s'est déroulé en France métropolitaine. Il s'agit d'abord du décalage culturel important entre ces juifs vivant en terre d'Islam et la France des Lumières : façonnés par des siècles de cohabitation avec les musulmans, les juifs ont leur propre langue (le judéo-arabe), des us et coutumes proches de ceux des ethnies locales, et cela expliquera leurs résistances à la tutelle métropolitaine (ainsi les rabbins traditionnels garderont-ils longtemps leur influence sur les populations locales). Il s'agit ensuite de l'exigence de "régénération " (transformation des mours et des modes de vie) posée en préalable à toute attribution de droits égaux : une autre logique d'émancipation a donc été appliquée ici, de même qu'aux juifs alsaciens lors du décret infâme de 1808, contraire à l'esprit libéral des premiers révolutionnaires.

Si les mêmes réformes religieuses ont bien été réalisées, notamment l'imposition d'une hiérarchie religieuse là où chaque communauté locale avait son autonomie, et la distinction entre laics et ecclésiastiques (via les rabbins métropolitains importés), cela ne s'est pas fait sans résistances, surtout passives, qui auraient duré jusqu'au lendemain de la Première Guerre mondiale. On peut s'étonner, si l'on compare cette analyse à celle de Joëlle Allouche-Benayoun sur le même sujet (2006), de voir ici tant d'insistance sur ces résistances, et quasiment aucun développement sur les inégalités et les difficultés de la condition de dhimmi (protégé). Celle-ci est certes mentionnée mais elle est mise en regard, par Nadjari, de la plus grande indépendance communautaire préservée de la sorte. De même, l'auteur n'évoque pas les marques de sympathie des juifs d'Algérie pour ces colonisateurs qui pourraient les sortir de la condition de dhimmi (nombreuses selon Allouche-Benayoun). Les deux auteurs ne sont pas d'accord non plus sur la persistance des lieux de culte privés (sous l'autorité d'un chef de clan), restés nombreux pour Nadjari alors que Allouche-Benayoun parle de leur disparition progressive, ni sur le moment d'émergence d'une élite locale acquise au modèle juif français d'émancipation (lendemain de la Première Guerre mondiale selon Nadjari, bien avant selon Allouche-Benayoun). 
Si l'on compare maintenant l'article de Nadjari aux paragraphes consacrés par Leff aux rapports entre juifs de France et d'Algérie, on ne peut qu'être frappé de la démarche strictement compréhensive (selon la méthodologie wébérienne) de Leff qui met en avant les intentions positives des premiers à l'égard des seconds, alors que Nadjari adopte d'emblée un langage critique (au sens premier du terme) qui situe bien sa posture dénonciatrice. C'est toute la question du privilège accordé aux intentions des acteurs et aux représentations idéologiques de l'époque (au risque de l'angélisme) ou du choix d'une posture critique à partir de critères moraux, politiques et idéologiques d'aujourd'hui (au risque de l'anachronisme dans ce $\mathrm{cas}^{22}$ ).

\section{Les transformations du franco-judaïsme : recompositions ou ruptures?}

Les deux ouvrages historiques et le dossier proposés (dont les études sont historiques et/ou de philosophie politique) proposent toute une série d'analyses sur les caractéristiques du franco-judaisme et sur leurs transformations. Ils suscitent alors inévitablement la question du degré de plasticité de cette catégorie et celle du seuil au-delà duquel il faudrait éventuellement considérer l'ampleur des transformations comme une rupture (Bordès-Benayoun, 2004 ; Charbit, 2006) ${ }^{23}$.

\section{Le judaïsme : entre les apories de l'universalisme et celles du particularisme}

Dans son chapitre sur «la redéfinition du franco-judaïsme » entre 1920 et 1932 (Reshaping Franco-Judaism), Nadia Malinovich situe les transformations qu'elle a observées par rapport aux discours des leaders religieux et intellectuels juifs au XIX ${ }^{\mathrm{c}}$ siècle. Ceux-ci avaient à cœur, dit-elle, de démontrer la contribution des juifs à la culture occidentale et la compatibilité du judaisme avec l'universalisme (celui de la culture française en particulier); quant aux juifs français des années vingt, le centre de gravité de leur discours se serait déplacé : ils soulignaient plutôt leur particularisme juif, mais en argumentant bien de la non contradiction entre cette appartenance et l'engagement universaliste. Le déplacement est subtil certes, mais il attesterait selon nous d'une première inflexion : une conception de l'universel plus ouverte aux particularismes, véritablement

22. Lorsque D. Nadjari écrit, à propos du décret Crémieux de 1870 : «La III République naissante n'avait fait là que parachever le grand dessein que la France révolutionnaire et napoléonienne avait conçu pour "ses Juifs» (p. 77), on peut se demander si la France avait vraiment un " grand dessein " pour "ses Juifs".

23. Je discuterai ici de la thèse de Chantal Bordès-Benayoun sur la persistance d'un modèle " israélite " par delà ses variations. Je ferai également référence à l'article de Denis Charbit qui porte sur la période du «Réveil » et jusqu'à nos jours (plus brièvement). 
inclusive de leur diversité, là où la précédente serait plus uniformisante - réduisant les singularités à des phénomènes contingents. Ou " secondaires ». Ainsi les réinterprétations des thèmes de la tradition religieuse par les juifs du XIX ${ }^{\mathrm{e}}$ siècle, si l'on suit la thèse de Lisa Moses Leff, visaient bien à légitimer la nouvelle appartenance à la France, qui devenait première dans l'ordre politique, lequel était lui-même englobant de l'ordre religieux (Cohen, 1994), alors que les juifs des années vingt faisaient appel aux valeurs et à la culture françaises pour légitimer le particularisme juif (Malinovich : 206).

Mais le particularisme juif doit-il être défendu en étant mis sur le même plan que les autres particularismes? La discussion peut être historique et politique ou philosophique et politique (Milner, Levinas, Lévy). Malinovich évoque par exemple (pp. 203-207) les comparaisons faites par des écrivains pro-sionistes des années vingt entre sionisme et identités régionales. La valorisation des différences régionales était alors un thème de gauche et, pour ceux qui n'étaient pas juifs, l'équation entre identité juive et régionalisme permettait de soutenir une vision ethnique du judaïsme (et même l'objectif sioniste) sans nier l'intégration des juifs en France, en se distinguant donc des discours antisémites qui ne visaient qu'à expulser les juifs ${ }^{24}$. La comparaison avec la situation nord-américaine se révèle ici pertinente : si la référence à un autre pays, pour un juif américain comme pour les autres immigrés de ce pays, impliquait pour tous une localisation extraterritoriale, en France, poursuit l'auteure, la « région » alors promue par les juifs n'était pas intérieure à la France comme l'étaient de fait la Bretagne ou la Provence; et cet argument pouvait donc aisément être retourné contre les juifs. De la même manière, la valorisation de la différence juive, dans le contexte de développement d'un nationalisme français de type ethnique, risquait de radicaliser (essentialiser) l'altérité des juifs - à l'instar des Noirs par exemple - mettant en cause "le statut des juifs comme résidents de longue date en France, dont l'existence était liée à leur statut politique de citoyens pleinement reconnu comme tels» (Malinovich : 194).

Tous les particularismes ne pourraient donc être mis sur le même plan - d'autant que, comme le note Malinovich, la distinction ténue entre célébration de la différence et renforcement des stéréotypes racistes pouvait être aisément effacée $^{25}$. Cette remarque nous permet de rejoindre le questionnement de Milner à propos de "l'universel illimité ", sans bornes, qui ne serait ouvert aux minorités qu'en les juxtaposant, c'est-à-dire en les uniformisant finalement, accumulant

24. N. Malinovich cite le discours de Justin Godart, membre non juif de l'association France-Palestine : «Si le mouvement sioniste réussissait, nous ne souhaitons pas mettre en question la profonde assimilation des juifs français, ou douter de leur patriotisme, ou l'affaiblir en leur reprochant d'avoir deux patries" (cité p. 204, traduit par moi, sans vérification de la citation originale en français).

25. L. M. Leff a elle aussi décrit le retournement de l'internationalisme juif par ses adversaires, d'un universalisme positif en une vision négative du " complot juif ». 
leurs « droits » comme si la majorité n'était qu'un amas de minorités (on reprend ici les analyses et formulations de Savy et Schreiber dans leur article de clôture du dossier). S'il faut ainsi préserver la singularité de chaque groupe comme de chaque personne, faut-il pour autant accorder au " nom propre "Juif » une singularité plus particulière, comme le propose Milner ? Savy et Schreiber posent la question et ne semblent pas vouloir le suivre sur ce point. Si le paradigme universaliste a ainsi façonné les modes d'expression du particularisme juif ${ }^{26}$, soit en réduisant sa différence à une contingence peu signifiante au sein d'un universel global, soit en assimilant totalement cette différence aux autres particularismes, l'insistance sur la singularité doit-elle " absolutiser » celle des juifs ? L'option particulariste n'est donc pas exempte, elle aussi, de contradictions.

Remarquons, pour finir sur ce point, que Birnbaum s'oppose à la vision négative de Milner à propos des États-Unis, qui seraient pour ce dernier l'incarnation contemporaine d'un universel illimité (Savy-Schreiber, p. 120), alors que l'historien y voit un espace ouvert au pluralisme et la possibilité d'une vie juive riche, grâce à la pluri-appartenance.

\section{Le franco-judaïsme : jusqu'où le modèle peut-il se décliner sans changer de nature?}

Nadia Malinovich conclut son étude en précisant ce qu'elle entend par définition « ethnoculturelle " de l'identité juive : soit une perspective nationale comme le sionisme (d'autres versions sont possibles), soit une vision religieuse mais où le particularisme juif est valorisé ; tout en soulignant le caractère minoritaire de cette vision parmi les juifs, elle s'interroge sur la diffusion de ce modèle dans la société française dans son ensemble. Elle introduit dans la discussion l'argument de Catherine Fhima (2006), selon qui la vision religieuse du judaïsme serait restée alors prévalente, d'autant que la gauche laique de l'époque aurait eu du mal à imaginer un juif français pleinement moderne et ethnique ${ }^{27}$.

La question se pose en effet : peut-on envisager un tel passage vers «l'ethnoculturel » dans une société qui a toujours eu tendance, si ce n'est à éradiquer les particularismes, au moins à les secondariser (Chanet, 1994) ? Si des courants de valorisation de la différence ont périodiquement surgi (à gauche comme à droite d'ailleurs), n'est-ce pas dans le degré d'insistance sur cette différence - ou de diffusion sociale de cette valeur - et dans le rapport établi entre cette différence et le « tout » de la nation (inclusion, complémentarité, ou exclusion), que résiderait le subtil équilibre de ce « franco-judaïsme ».... ou sa rupture ?

Le " découpage en domaines " lié à la confessionnalisation et tant décrié par plusieurs auteurs de la revue Labyrinthe (ou par les "penseurs du retour ») a été également contesté par d'autres juifs au XIX ${ }^{\mathrm{e}}$ siècle et dans les années vingt.

26. Les analyses des deux historiennes se rejoignent sur ce point.

27. Cité par N. Malinovich, p. 238. 
Leur contestation s'est traduite soit par l'affirmation d'une identité juive «intégrale " (pour prendre un terme analytique contemporain), au plan religieux comme au plan séculier avec le sionisme - sans que cet intégralisme implique forcément séparatisme ou refus de la société globale, notons-le - soit par la revendication d'une diversité légitime des manières d'être juif, parmi lesquelles peuvent figurer toutes les formules culturelles et «nationales " d'une identité juive séculière. Le "subtil équilibre du "franco-judaïsme" dont nous parlions plus haut aurait été ainsi préservé dans le cas de ces leaders juifs français du $\mathrm{XIX}^{\mathrm{e}}$ siècle qui affichaient leur identité juive dans l'arène politique (sans la « discrétion» et la privatisation de l'identité juive que l'on associe généralement à «l'israélite »), mais c'était alors pour mieux défendre la France et ses idéaux à travers le monde (selon l'étude de Leff). L'équilibre aurait été préservé également dans le cas de ces écrivains juifs français pro-sionistes (André Spire, Albert Cohen, ...) qui n'envisageaient la création d'un foyer national que pour les juifs persécutés dans le monde, c'est-à-dire pas pour les juifs français, en raison de leur bonne intégration ${ }^{28}$. Mais ce "subtil équilibre" serait-il maintenu dans le cas, par exemple, d'un Benny Lévy affichant son « étrangeté » de juif par rapport à la France ? Cette forme "exclusive " d'intégralisme religieux ne contredit-elle pas ce qui serait le cœur (ou l'un des traits centraux) du franco-judaïsme : la symbiose revendiquée entre judéité et francité ? De même, si bien des formules de compatibilité peuvent être élaborées entre soutien au sionisme ou à Israël et francité - jusqu'à cet étonnante revendication d'un jeune juif d'aujourd'hui : " sioniste et citoyen ${ }^{29}$ - peut-on dire d'un juif français qui déciderait de s'installer en Israël qu'il reste dans le cadre du franco-judaïsme ? Certes on peut toujours repérer des filiations idéologiques ou des affinités, même paradoxales, mais ne doit-on pas limiter l'extension définitionnelle du terme pour mieux assurer sa pertinence?

L'on peut donc accepter l'hypothèse de la sociologue Chantal BordèsBenayoun sur la plasticité de la catégorie "israélite " - équivalente pour elle à celle de "franco-judaïsme »- et sur la pluralité de ses expressions possibles, y compris les « juifs à trait d'union » évoqués par Annie Kriegel ${ }^{30}$; l'on peut aussi admettre avec elle que le franco-judaïsme n'a pas disparu, au contraire de ce que prétendraient les tenants d'une affirmation identitaire forte contre une image volontairement édulcorée de l'israélite. Mais il nous faut alors " poursuivre le bilan historique » que Bordès-Benayoun appelle de ses vœux, et évaluer comment « les termes du franco-judaïsme ont changé » (p. 41).

28. Selon notre analyse, le titre de l'article de Denis Charbit: «Déclinaisons du francojudaïsme " serait ainsi tout à fait pertinent (art. cit.).

29. Où « citoyen " se décline ici prioritairement comme « citoyen français », avec une forte insistance sur les valeurs françaises comme sources même d'un soutien à Israël et au sionisme. Voir l'article de Paul Bernard, 2003. (Paul Bernard était président de l'UEJF peu avant cette publication).

30. Cité par C. Bordès-Benayoun, p. 39. 
Les réflexions suscitées par les travaux présentés ici (les deux ouvrages historiques notamment) et nos propres travaux sur les affirmations identitaires juives en France depuis les années soixante-dix, nous poussent à proposer maintenant quelques hypothèses complémentaires sur les caractéristiques du franco-judaïsme qui nous semblent les plus pertinentes.

En partant de l'idée de "symbiose " entre judéité et francité - c'est le terme des acteurs juifs - et en observant la diversité de ses expressions, confessionnelles, culturelles, ethniques ou nationales, on pourrait poser (comme on l'a écrit plus haut) que le degré de valorisation de la différence et le rapport posé entre le judaisme et le " tout » de la nation sont susceptibles de déterminer la « compatibilité » entre les deux termes de l'identité, ou au contraire leur rupture (leur étrangeté, pour reprendre le terme de Benny Lévy). Ainsi, qu'il y ait ou non insistance sur le particularisme juif, que celui-ci soit de nature confessionnelle ou séculière, culturelle ou politique, on resterait dans les limites $d u$ francojudaïsme si le rapport de cette identité juive à la francité est d'inclusion (secondarisation) ou de complémentarité (sur un même plan d'égalité). Toute exclusion d'un terme par l'autre (qu'il s'agisse de la politique raciale de Vichy ou de l'intégralisme de Lévy) impliquerait une rupture du modèle.

Reconnaissons qu'il s'agit là d'une vision peu canonique de la conception de la nation française, par rapport à l'image communément admise de sa tradition politique. Mais n'est-ce pas là la leçon qu'il faut tirer des études historiques ? Pourtant il nous faut aussi tenir compte de cette « image communément admise ", c'est-à-dire de ces mémoires historiques persistantes sans cesse mises en jeu dans les périodes conflictuelles (et éventuellement qualifiées de «tradition politique»). N'est-ce pas cette "mémoire » d'un État fort et protecteur - malgré certains démentis historiques rudes - qui incite certains auteurs du dossier de Labyrinthe à se replier brusquement sur un "modèle républicain » au lieu de tenter d'imaginer une version française de la vitalité juive américaine ou allemande ? Aucune des transformations contemporaines de l'État français - de la décentralisation à la construction européenne en passant par le recours aux acteurs de la «société civile », y compris des acteurs religieux - n'auraient donc d'impact sur cet État «fort»?

Évoquons pour terminer un aspect non encore abordé dans ces discussions : la dimension institutionnelle des expressions publiques du judaïsme français. Celle-ci joue certainement un rôle dans la perception des juifs, notamment dans l'ordre politique. De ce point de vue, la différence majeure entre le "Réveil » des années vingt et les affirmations identitaires juives contemporaines réside dans le fait de leur traduction institutionnelle, depuis la fin de la Seconde Guerre mondiale, avec la création du CRIF en 1943-44 - même si cette institution de représentation "politique » des juifs est restée sans impact véritable jusqu'aux années soixante-dix - puis la création en 1949 d'une association fédérative à vocation sociale et culturelle, le FSJU, dont l'importance ira croissante aussi à 
partir de ces mêmes années. La montée en puissance de ces institutions considérées comme "représentatives» par l'État aux plans politique et culturel, aux côtés d'une institution consistoriale toujours considérée comme représentative au plan religieux (malgré son affaiblissement récent), traduit le fait que la pluralisation des identités juives est devenue un trait dominant de la vie juive en France (Mandel, $2003: 318)^{31}$. Pour autant, si le "modèle confessionnel " se trouve ainsi débordé ${ }^{32}$, la " politisation » de cette vie juive et surtout son institutionnalisation ne constituent-elles pas un passage à la limite et peut-être une rupture par rapport au franco-judaïsme? Si cette institutionnalisation traduit une défiance (relative ou conjoncturelle) par rapport à l'État, comme le souligne Pierre Birnbaum dans ses essais récents (1995), cela implique-t-il forcément une défiance plus générale par rapport à la société française et donc une mise en question de la «compatibilité » entre fidélité juive et citoyenneté française ?

\section{"Complexifier les Lumières "}

$S$ 'il faut " complexifier les Lumières » (question des coordinateurs de la revue à Birnbaum, à laquelle il ne répond pas vraiment), on peut appeler aussi à penser de manière complexe l'identité juive, sans la figer ou l'essentialiser. Les auteurs critiques évoqués dans la revue Labyrinthe n'auraient-il pas en tête une "vie juive » dont la plénitude serait mythique - ou n'existerait qu'au regard du manque ressenti aujourd'hui - comme une nostalgie pour un autre espace-temps, même lorsqu'ils en connaissent les aspects sombres ou dramatiques? Les historiennes Lisa Leff et Nadia Malinovich militent dans le sens de cette complexité et d'une conception non fixiste de l'identité juive, dont elles exposent les mille et une recompositions, à travers les réinterprétations de la tradition. Elles prolongent également les réévaluations historiques du processus d'émancipation des juifs, en montrant que l'intégration fut loin d'impliquer une disparition pure et simple de l'identité et que même l'«assimilation » ne fut pas sans accompagner paradoxalement, parfois, une valorisation publique de l'identité juive.

Martine COHEN

Paris, CNRS-EPHE, Groupe Sociétés, Religions, Laïcités martine.cohen@gsrl.cnrs.fr

31. M. Mandel évoque dans son étude comparative, la montée d'une conception ethnique des liens entre les juifs dans le monde (qui serait devenue "mainstream»), et la légitimation croissante de la diversité des expressions identitaires juives.

32. Voir l'un des articles où l'on développe cette thèse : "Les Juifs de France. Modernité et identité ", Vingtième Siècle. Revue d'Histoire, 66, avril-juin 2000, pp. 91-106. 


\section{Bibliographie}

AlberT Phyllis Cohen, 1977, The Modernization of French Jewry: Consistory and Community in the Nineteenth-Century, Hanover (NH), Brandeis University Press.

Allouche-Benayoun Joëlle, 2006, "Les enjeux de la naturalisation des Juifs d'Algérie ", in Luizard P.-J., (dir.), Le choc colonial et l'islam. Les politiques religieuses des puissances coloniales en terres d'islam, Paris, La Découverte, pp. 179-195.

Bensoussan Georges, 2006, L'Europe, une passion génocidaire. Essai d'histoire culturelle, Paris, Les Mille et Une Nuits.

Bernard Paul, 2003, "Sioniste et citoyen ", dans Le Sionisme expliqué à nos potes, publication collective de l'Union des Étudiants Juifs de France, Paris, Éditions de La Martinière, pp. 163-189.

Birnbaum Pierre, 1995, Destins juifs. De la Révolution française à Carpentras, Paris, Calmann-Lévy.

-, 2007, L'Aigle et la Synagogue. Napoléon, les Juifs et l'État, Paris, Fayard.

BORDĖs-BENAYOUN Chantal, 2004, "Variations et actualité du modèle israélite ", in P. Cabanel, C. Bordès-Benayoun, Un modèle d'intégration, juifs et israélites en France et en Europe, $\mathrm{XIX}^{e}-\mathrm{XX} \mathrm{X}^{e}$ siècles, Paris, Berg International, pp. 31-41.

Chanet Jean-François, 1994, L'École républicaine et les petites patries, Paris, Aubier.

Charbit Denis, 2006, "Déclinaisons du franco-judaïsme ", in Biale D., (dir.), Les Cultures des Juifs. Une nouvelle histoire, Paris, Éditions de l'Éclat, pp. 1003-1042.

CoHEN Martine, 1984, "De l'émancipation à l'intégration: les transformations du judaïsme français au XIX ${ }^{c}$ siècle ", Archives de Sciences Sociales des Religions, 88, pp. 5-22.

-, 2000, «Les Juifs de France. Modernité et identité », Vingtième Siècle. Revue d'Histoire, 66, avril-juin 2000, pp. 91-106.

COHEN Monique-Lise, 1992, Les Juifs ont-ils du cour ? Discours révolutionnaires et antisémitisme, Toulouse, Vent Terral.

FHIMA Catherine, 2006, " Au cœur de la "renaissance juive" des années 1920 : littérature et judéité ", Archives juives, 39-1, 2006, pp. 29-45.

Levinas Emmanuel, 1963 (2 éd. 1994), Difficile liberté. Essais sur le judaïsme, Paris, Albin Michel.

Mandel Maud, 2003, In the Aftermath of Genocide. Armenians and Jews in TwentiethCentury France, Durham-London, Duke University Press.

Marrus Michacl, 1971, The Politics of Assimilation. A Study of the French Jewish Community at the time of the Dreyfus Affair, Oxford University Press [Les Juifs de France à l'époque de l'affaire Dreyfus. L'assimilation à l'épreuve, Paris, CalmannLévy, 1972].

Meschonnic Henri, 2001, L’Utopie du Juif, Paris, Desclée De Brouwer.

Michel Alain, 2003, Juifs, Français et Scouts. L'bistoire des EI de 1923 aux années 1990, Jérusalem, Éditions Elkana.

THIEsSE Anne-Marie, 1988, "Le Mouvement littéraire régionaliste, 1900-1945 », Ethnologie française, 18-3, pp. 220-232.

Trigano Shmuel, 2006, L'avenir des Juifs de France, Paris, Grasset. 